\title{
Sharp form of inequality for the constant $e$
}

\section{ChaO-PING CHEN and CRISTINEL MORTICI}

\section{ABSTRACT.}

(i) We determine the best possible constants $\alpha$ and $\beta$ such that the inequalities

are valid for all integers $n \geq 1$.

$$
2(n+\alpha)\left(\frac{2^{n} n !}{(2 n) !}\right)^{1 / n}<e \leq 2(n+\beta)\left(\frac{2^{n} n !}{(2 n) !}\right)^{1 / n}
$$

(ii) Let the sequence $v_{n}$ be defined by

$$
v_{n}=2\left(n+\frac{\ln 2}{2}+\frac{a}{n}+\frac{b}{n^{2}}\right)\left(\frac{2^{n} n !}{(2 n) !}\right)^{1 / n} .
$$

We determine the values $a, b$ which provide the fastest sequence $\left(v_{n}\right)_{n \geq 1}$ approximating the constant $e$.

HeNAN POLYTECHNIC UNIVERSITY

SCHOOL OF MATHEMATICS AND INFORMATICS

Jiaozuo City 454003, HenAn Province, China

E-mail address: chenchaopingesohu.com

VALAHIA UNIVERSITY OF TÂRGOVIŞTE

DEPARTMENT OF MATHEMATICS

UNIRII 18, 130082 TÂRGOVIŞTE, ROMANIA

E-mail address: cmorticievalahia.ro

Received: 01.10.2010; In revised form: 28.05.2011; Accepted: 30.06.2011

2010 Mathematics Subject Classification. 11Y60, 33B15.

Key words and phrases. Constant e, inequality, gamma function, psi function, polygamma functions, asymptotic formula. 